\title{
Aplicação de gesso e calcário na recuperação de solos salino-sódicos do Estado de Pernambuco ${ }^{1}$
}

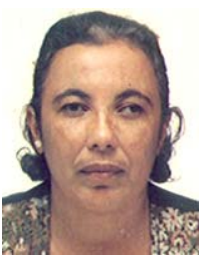

Maria de F. C. Barros ${ }^{2}$, Maurício P. F. Fontes ${ }^{3}$, Víctor H. Alvarez V. ${ }^{3}$ \& Hugo A. Ruiz ${ }^{3}$

1 Parte da Tese de Doutorado do primeiro autor, apresentada à Universidade Federal de Viçosa UFV
2 UFRPE/ DEPA, CEP 52171-900. Recife, PE. Fone: (81) 3302-1220. E-mail: fatimacb@ufre.br
${ }^{3}$ UFV/DS, CEP 36571-000, Viçosa, MG. Fone: (31) 3899-1057, E-mail: mpfontes@mail.ufv.br, vhav@mail.ufv.br,
hruiz@mail.com.br

Protocolo 137 - 23/10/2003 - Aprovado em 30/3/2005

\begin{abstract}
Resumo: Experimento em colunas de solo foi conduzido, objetivando-se avaliar o efeito da aplicação de gesso e gesso + calcário, na recuperação de solos salino-sódicos do Perímetro Irrigado de Custódia, PE. Os tratamentos foram dispostos em um delineamento em blocos casualizados, com arranjo fatorial de quatro solos, dois métodos de aplicação de gesso e gesso + calcário (aplicados na superfície e incorporados aos primeiros $5 \mathrm{~cm}$ da coluna de solo), duas combinações dos corretivos $(100 \%$ de gesso $+0 \%$ de calcário e $80 \%$ de gesso $+20 \%$ de calcário), calculados com base na necessidade de gesso dos solos, e quatro faixas de granulometria de gesso $(2,0-1,0,1,0-0,5,0,5-0,3$ e $<0,3 \mathrm{~mm})$, com três repetições. A quantidade de gesso determinada em laboratório pelo método de Schoonover $\mathrm{M}-1$, mostrou-se adequada no deslocamento do sódio trocável do complexo de troca. A eficiência do gesso e da mistura gesso + calcário na recuperação dos solos revelou-se superior quando os corretivos foram incorporados aos primeiros $5 \mathrm{~cm}$ das colunas de solo. Entre as granulometrias de gesso, as frações mais finas (0,5-0,3 mm e < 0,3 mm) apresentaram melhor desempenho na substituição do sódio trocável do complexo de troca.
\end{abstract}

Palavras-chave: sódio trocável, sodicidade, necessidade de gesso

\section{Application of gypsum and limestone in the reclamation of saline-sodic soils of the Pernambuco State}

\begin{abstract}
An experiment was carried out in soil columns with the objective of evaluating the effect of application of gypsum and limestone on reclamation of the saline-sodic soils in the Irrigation district of Custódia, PE. The treatments were arranged in a randomized block design in a factorial scheme of four soils, two methods for applications of gypsum and gypsum plus limestone (applied on surface and incorporated into the first five $\mathrm{cm}$ of the soil column), two combinations of the chemical amendments (100\% gypsum plus $0 \%$ limestone and $80 \%$ gypsum plus $20 \%$ limestone), calculated on the basis of gypsum requirement of soil and four granulometry gypsum strips $(2.0-1.0 ; 1.0-0.5 ; 0.5-0.3$ and $<0.3 \mathrm{~mm})$ with three replications. The gypsum amount determined by the method of Schoonover $\mathrm{M}-1$, under laboratory conditions, shows to be adequate in the displacement of the exchangeable sodium of the soil exchange complex. The efficiency of the gypsum as well as the gypsum plus lime mixture in reclamation of the soils shows to be superior, when the amendments are incorporated into the first $5 \mathrm{~cm}$ of the soil columns. Among the gypsum granulometry, the finest fractions, $(0.5-0.3 \mathrm{~mm}$ and $<0.3 \mathrm{~mm}$ ), presented better performance in replacing the exchangeable sodium of the exchange complex.
\end{abstract}

Key words: exchangeable sodium, sodicity, gypsum requirement

\section{INTRODUÇÃO}

Solos afetados por sais ocorrem extensivamente sob condições naturais, mas os maiores problemas de salinização são representados por solos anteriormente produtivos, que se tornam salinos devido ao manejo inadequado da irrigação. A alta salinidade e o elevado teor de sódio trocável desses solos são fatores limitantes à sua produtividade gerando, com isto, sérios problemas de ordem econômica, pois tais solos se tornam inaptos para agricultura, sendo então descartados do sistema de produção. 
A correção dos solos salino-sódicos e sódicos requer que o excesso de sódio trocável seja substituído pelo cálcio e que o produto dessa reação seja removido da zona radicular, por lixiviação.

Por apresentar menor custo, fácil manuseio e relativa facilidade com que é encontrado no mercado, quando comparado a outros corretivos, o gesso é o produto mais utilizado para correção de solos salino-sódicos (Pereira et al., 1986; Alcordo \& Rechcigl, 1993; Qadir et al., 1996; Holanda et al., 1998; Ramirez et al., 1999). O gesso pode ser encontrado como minério (gesso de jazidas) ou como subproduto da indústria de fertilizantes (gesso agrícola). O Estado de Pernambuco possui $83 \%$ das reservas de gesso de jazidas do País, localizadas próximo aos perímetros irrigados (Magalhães, 1995). A quantidade do corretivo, necessária à recuperação de solos salino-sódicos ou sódicos, pode ser determinada por método de laboratório ou calculada em função da percentagem de sódio trocável (PST) e da capacidade de troca de cátions (CTC).

A eficiência do gesso depende de sua dissolução, a qual é influenciada por diversos fatores, principalmente pela forma de aplicação e pela granulometria do corretivo.

A utilização de calcário também constitui alternativa para recuperação de solos com problemas de sodicidade, quando os solos apresentam $\mathrm{pH}<7,5$ e não contêm carbonatos (Richards, 1954). Devido à sua baixa solubilidade, o calcário tem sido pouco recomendado e seu efeito se restringe sobretudo em aumentar a concentração de eletrólitos: as trocas iônicas $\mathrm{Na}-\mathrm{Ca}$ ocorrem de forma mais lenta (McKenzie et al., 1995). De acordo com esses autores, as vantagens da combinação de gesso e calcário para correção de solos sódicos, podem ser resumidas em: a) garantir um imediato efeito sobre a concentração de eletrólitos e PST (efeito do gesso) e b) manter uma concentração eletrolítica necessária, por um longo período de tempo (efeito do calcário). Esses autores citam, ainda, a necessidade de futuras pesquisas para determinar o tamanho ótimo das partículas de gesso e calcário e as proporções ideais de calcário e gesso, na recuperação de solos com problemas de sodicidade.

Objetivou-se, neste trabalho, avaliar a eficácia do gesso aplicado ao solo em função da estimativa de laboratório no deslocamento do sódio trocável do complexo de troca, testar a influência do tamanho das partículas e do método de aplicação do gesso e do gesso + calcário, nas propriedades químicas de solos salino-sódicos.

\section{MATERIAL E MÉTODOS}

O ensaio em coluna de solo foi instalado no Laboratório de Salinidade do Solo da Universidade Federal Rural de Pernambuco. Para este estudo foram coletadas quatro amostras superficiais $(0-30 \mathrm{~cm})$ de solos salino-sódicos ( $\mathrm{S} 1, \mathrm{~S} 2$, S3 e S4), no perímetro irrigado de Custódia, no Estado de Pernambuco. Depois de coletadas, as amostras foram secadas ao ar, destorroadas e passadas em peneira de $2 \mathrm{~mm}$ de malha. Antes desse processamento retiraram-se torrões, para obtenção da densidade do solo. A pasta saturada foi preparada seguindose a metodologia descrita por Richards (1954) e no extrato da pasta saturada foi determinada a condutividade elétrica (CE).

Aplicando-se, ainda, a metodologia sugerida por Richards (1954), determinaram-se: o pH dos solos na relação solo-água $(1: 2,5)$ e a capacidade de troca de cátions (CTC), pelo método do acetato de sódio; o sódio trocável extraído com uma solução de acetato de amônio $1 \mathrm{~mol} \mathrm{~L}^{-1}$ a pH 7,0. A percentagem de sódio trocável (PST) foi obtida a partir dos dados da CTC e do sódio trocável, pela equação PST $=\left(\mathrm{Na}^{+} / \mathrm{CTC}\right) \times 100$.

A composição granulométrica foi determinada segundo a metodologia descrita pela EMBRAPA (1997), após lavagem com etanol a $60 \%$, até a eliminação total de cloretos. O equivalente de carbonato de cálcio, por titulação, e o gesso por precipitação (Richards, 1954). A densidade do solo foi determinada pelo método do torrão parafinado (EMBRAPA, 1997). As características químicas e físicas das amostras de solo encontram-se na Tabela 1.

Os tratamentos foram dispostos em um delineamento em blocos casualizados, com arranjamento fatorial $4 \times 2 \times 2 \times 4$ (quatro solos, dois métodos de aplicação, duas porcentagens de gesso e calcário e quatro granulometrias), com três repetições, totalizando 192 unidades experimentais.

A necessidade de gesso determinada pelo método de Schoonover M-1 (Barros \& Magalhães, 1989), foi aplicada ao solo pela combinação de gesso e calcário, de acordo com os seguintes tratamentos: $100 \%$ gesso $+0 \%$ calcário e $80 \%$ gesso $+20 \%$ calcário. Utilizaram-se dois métodos de aplicação; primeiro, o gesso e o gesso + calcário foram aplicados uniformemente sobre a superfície de cada coluna de solo; na segunda metodologia, o gesso e o gesso + calcário foram incorporados aos primeiros $5 \mathrm{~cm}$ de solo, antes do acondicionamento na coluna.

As frações granulométricas do gesso utilizadas foram: partículas com diâmetro entre 2,0 e 1,0 mm (G1), partículas com diâmetro entre 1,0 e $0,5 \mathrm{~mm}(\mathrm{G} 2)$, partículas com diâmetro entre 0,5 e $0,3 \mathrm{~mm}(\mathrm{G} 3)$, e partículas com diâmetro $<0,3 \mathrm{~mm}(\mathrm{G} 4)$. A

Tabela 1. Características químicas e físicas dos solos

\begin{tabular}{|c|c|c|c|c|c|c|c|c|c|c|c|c|c|}
\hline \multirow{3}{*}{ Solo } & \multicolumn{4}{|c|}{ Cátions Trocáveis } & \multirow{2}{*}{$\mathrm{NG}^{(1)}$} & \multirow{2}{*}{$\mathrm{CTC}^{(2)}$} & \multirow[b]{2}{*}{ Gesso } & \multirow{2}{*}{$\mathrm{PST}^{(3)}$} & \multirow{2}{*}{$\begin{array}{c}\mathrm{CaCO}_{3} \\
\text { equivalente }\end{array}$} & \multirow{3}{*}{$\mathrm{pH}$} & \multirow{3}{*}{$\begin{array}{c}\mathrm{CE}^{(4)} \\
\mathrm{dS} \mathrm{m}{ }^{-1}\end{array}$} & \multirow{3}{*}{ Classe Textural } & \multirow{3}{*}{$\begin{array}{c}\text { Densidade } \\
\text { do Solo } \\
\mathrm{g} \mathrm{cm}^{-3}\end{array}$} \\
\hline & $\mathrm{Ca}^{2+}$ & $\mathrm{Mg}^{2+}$ & $\mathrm{Na}^{+}$ & $\mathrm{K}^{+}$ & & & & & & & & & \\
\hline & \multicolumn{7}{|c|}{$\mathrm{cmol}_{\mathrm{c}} \mathrm{dm}^{-3}$} & \multicolumn{2}{|r|}{$\%$} & & & & \\
\hline S1 & 6,71 & 2,92 & 5,40 & 0,27 & 6,45 & 15,35 & 0,00 & 35,18 & 0,00 & 7,01 & 6,12 & Franco & 1,50 \\
\hline S2 & 7,08 & 2,12 & 6,23 & 0,32 & 6,98 & 15,80 & 0,00 & 39,43 & 0,00 & 7,17 & 8,38 & Franco argiloso & 1,50 \\
\hline S3 & 0,86 & 1,21 & 11,80 & 0,17 & 12,86 & 14,01 & 0,00 & 84,23 & 0,00 & 7,30 & 17,20 & Franco argilo arenoso & 1,60 \\
\hline S4 & 3,87 & 2,22 & 2,90 & 0,25 & 3,31 & 9,31 & 0,00 & 31,15 & 0,00 & 6,90 & 5,24 & Franco arenoso & 1,60 \\
\hline
\end{tabular}


granulometria do calcário empregada foi de partículas com diâmetro entre 1,0 e 2,0 mm.

As unidades experimentais foram constituídas de tubos de PVC, com $10 \mathrm{~cm}$ de diâmetro e $17 \mathrm{~cm}$ de altura. As amostras de solo foram acondicionadas nas colunas de PVC, de modo a se aproximarem da densidade do solo de cada amostra. Cada coluna de solo foi dividida em duas camadas, cada uma com 5 $\mathrm{cm}$ de espessura, correspondendo a $0,52 \mathrm{dm}^{3}$ de solo, tendo em sua base um sistema de drenagem. Inicialmente, as colunas de solo foram umedecidas lentamente, de cima para baixo, até atingirem a saturação; em seguida, as colunas de solo foram lixiviadas com água destilada, mantendo-se um nível constante de $2 \mathrm{~cm}$ acima da superfície do solo. Para a lixiviação utilizou-se uma lâmina de água equivalente a duas vezes o volume de poros, tendo sido aplicados $600 \mathrm{~mL}$. Durante o experimento, a temperatura foi mantida a $25^{\circ} \mathrm{C}$. Depois da lixiviação as colunas foram desmontadas e o solo de cada camada secado ao ar, destorroado e passado em peneira de $2 \mathrm{~mm}$ de malha determinando-se, em seguida, os cátions trocáveis, o gesso e o equivalente de carbonato de cálcio, de acordo com a metodologia descrita anteriormente.

Nas análises estatísticas utilizou-se o Sistema para Análises Estatísticas e Genéticas, realizando-se o teste de homogeneidade de variâncias, para cada variável. No presente trabalho foram consideradas variâncias homogêneas, quando o resultado do quociente entre o maior e o menor quadrado médio do resíduo para cada variável, entre os solos, era menor ou igual a seis.

Os dados obtidos foram interpretados por meio de análise de variância e de regressão, testando-se diversos modelos. Os critérios para escolha do modelo foram o maior valor do coeficiente de determinação ajustado e a significância dos coeficientes da equação de regressão.

\section{RESULTADOS E DISCUSSÃO}

Os resultados apresentados nas Figuras 1 e 2 indicam que, independentemente dos tratamentos utilizados, ocorreu grande diminuição do sódio trocável em relação aos valores originais, em especial na profundidade de $0-5 \mathrm{~cm}$. Esta tendência, embora com menor magnitude, é constatada também para a profundidade de $5-10 \mathrm{~cm}$, confirmando a eficiência da quantidade de gesso determinada em laboratório no deslocamento do sódio trocável do complexo de troca. O mesmo ocorre com a eficácia da aplicação de gesso de granulometrias diferenciadas e da mistura de gesso e calcário na recuperação dos solos salino-sódicos em estudo.

Para todas as amostras, os dados de sódio trocável indicam que os corretivos incorporados ao solo apresentaram-se mais eficazes que quando aplicados em superfície. Tal afirmativa está fundamentada no fato de ocorrer maior contato da água com as partículas de gesso e calcário, quando uma quantidade desses corretivos é incorporada à camada de $0-5 \mathrm{~cm}$ de profundidade, durante a lixiviação. Em virtude da baixa solubilidade do gesso, o tempo de contato da água com o corretivo é de importância fundamental para que ocorram maior solubilização, difusão e reações de troca entre o cálcio e o sódio.

Entre as granulometrias utilizadas, as partículas de maior superfície específica (G3 e G4) apresentaram-se mais eficazes no deslocamento do sódio trocável, cujo resultado se deve à maior solubilização e reatividade das partículas menores resultando, inicialmente, na maior concentração de cálcio em solução; este efeito é somado a uma substituição mais rápida do sódio trocável, enquanto nas partículas maiores (G1 e G2) a dissolução é relativamente mais lenta resultando, de início, em menor concentração de cálcio e menor substituição do sódio presente no complexo de troca.

Neste estudo, para todas as amostras estudadas, observase que as reações de troca entre o cálcio cedido pelos corretivos e o sódio trocável do solo foram bastante intensas quando se utilizaram partículas de menor tamanho, principalmente na camada de $0-5 \mathrm{~cm}$ de profundidade, semelhante ao ocorrido nos estudos de recuperação de solos sódicos em colunas de solo, realizados por Ahamad et al. (1997). Os autores observaram que a aplicação de gesso teve influência significativa na redução da PST do solo, decorrente da substituição de $96 \%$ do sódio trocável no complexo de troca. Valores extremamente baixos para sódio trocável depois da aplicação de gesso e lixiviação dos sais do solo, foram também relatados por Sampaio (1993) e Liang et al. (1995).

A aplicação em superfície foi menos eficaz na substituição do sódio trocável pelo cálcio e, no final do experimento, constatou-se a presença de gesso remanescente nos tratamentos que receberam gesso de diâmetro na faixa de 2,0-1,0 $\mathrm{mm}$, para as amostras $\mathrm{S} 1, \mathrm{~S} 2$ e S4. Com relação à amostra S3, por apresentar uma PST muito elevada (PST $>80 \%$ ), a maior quantidade de gesso requerida para que a recuperação fosse alcançada resultou em gesso remanescente em todas as faixas de granulometria utilizadas, tendo sido constatada, também, a presença de calcário (Tabela 2). Esses resultados indicam que, quando a amostra de solo apresentar uma PST $>40 \%$, uma lâmina maior que duas vezes o volume de poros deve ser utilizada para garantir que todo o gesso seja dissolvido e, conseqüentemente, mais cálcio esteja disponível para substituir o sódio no complexo de troca, aumentando assim, o sucesso da recuperação; entretanto, isto é somente uma conjectura, pois se ressente da necessidade da obtenção de dados experimentais que comprovem ou não esta hipótese. Tais resultados são similares aos encontrados por de Jong (1982), que não observou a presença de gesso quando este foi incorporado ao solo, mas quando o gesso foi aplicado em superfície, uma parte dele não foi dissolvida. $\mathrm{O}$ autor concluiu, também, que a menor performance do gesso aplicado na superfície é resultante da menor concentração de cálcio na água de percolação nessa forma de aplicação.

Nas Figuras 3 e 4 encontram-se os resultados da PST após aplicação dos tratamentos. Pode-se observar que todas as amostras de solo apresentaram a mesma tendência de maior diminuição da PST com o aumento da superfície específica das partículas de gesso e este efeito foi mais pronunciado nos tratamentos que receberam os corretivos incorporados aos primeiros $5 \mathrm{~cm}$ da coluna de solo. Os dados apresentados para 
A.

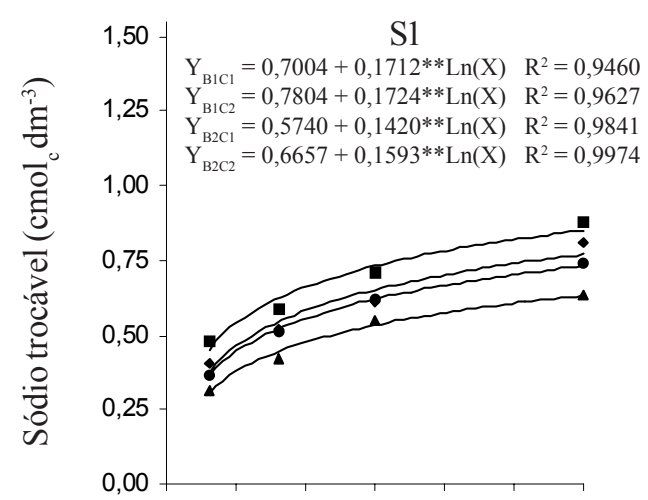

B.

C.

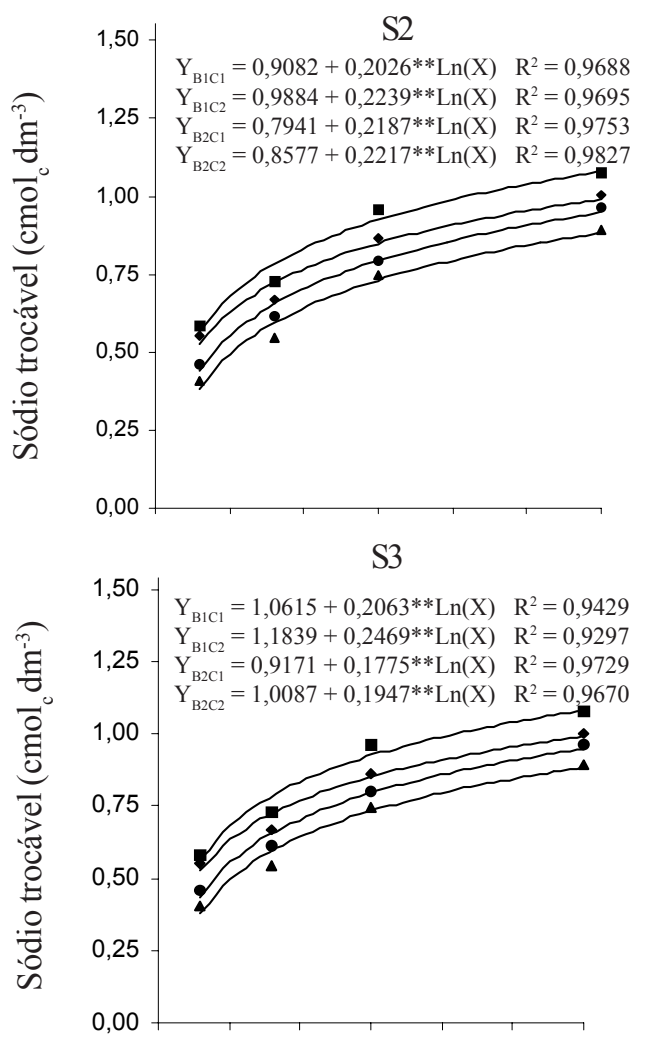

D.

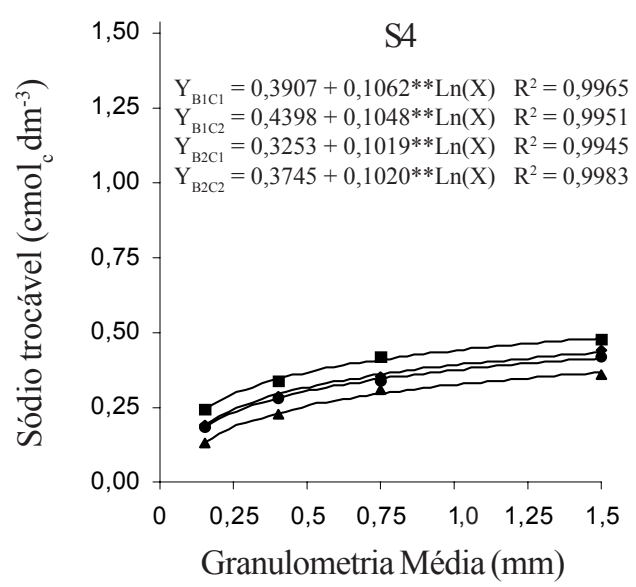

Figura 1. Sódio trocável na profundidade de $0-5 \mathrm{~cm}$ do solo, em relação à forma da aplicação dos corretivos na superfície (B1), incorporado (B2), mistura $\mathrm{C} 1$ (100\% de gesso $+0 \%$ de calcário) e mistura C2 (80\% de gesso $+20 \%$ de calcário), em função da granulometria média do gesso (G), G1 (1,5 mm), G2 (0,75 mm), G3 $(0,4 \mathrm{~mm})$ e G4 (0,15 mm), para os solos S1, S2, S3 e S4
A.

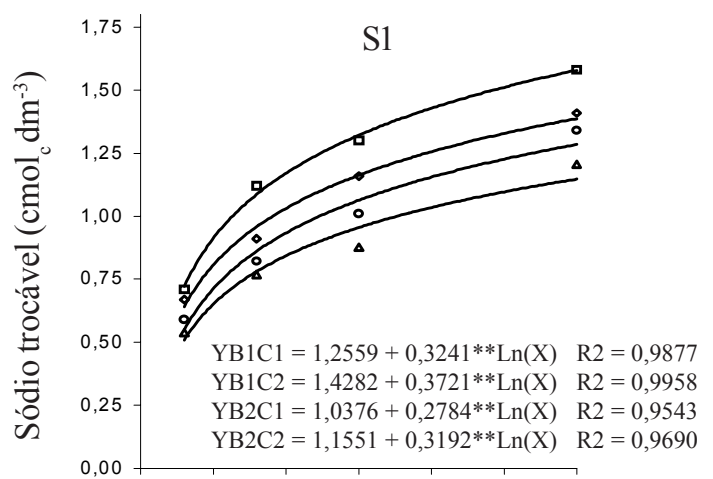

B.

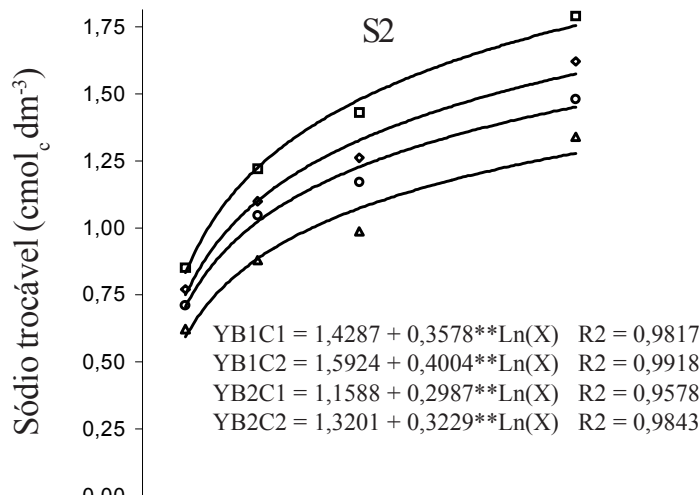

C.

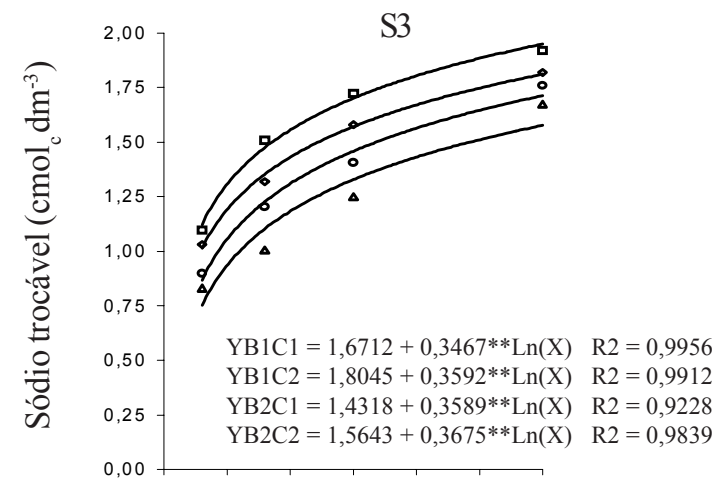

D.

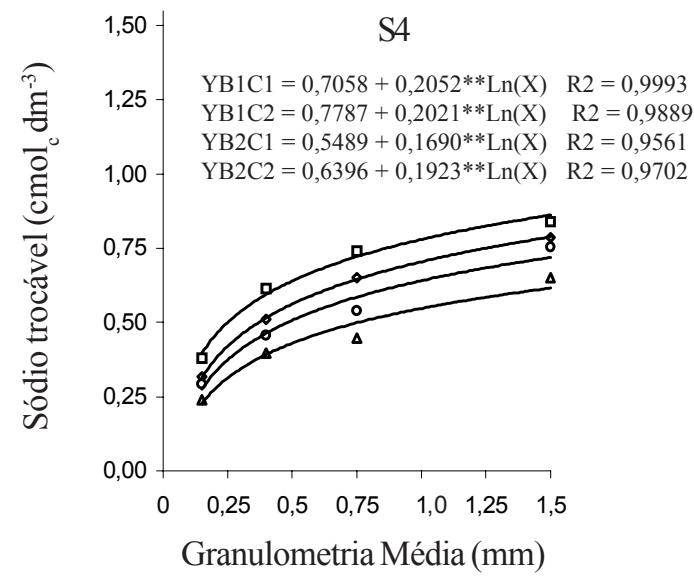

Figura 2. Sódio trocável na profundidade de $5-10 \mathrm{~cm}$ do solo, em relação à forma da aplicação dos corretivos na superfície (B1), incorporado (B2), mistura $\mathrm{C} 1$ (100\% de gesso $+0 \%$ de calcário) e mistura C2 (80 \% de gesso $+20 \%$ de calcário), em função da granulometria média do gesso (G), G1 (1,5 mm), G2 (0,75 mm), G3 $(0,4 \mathrm{~mm})$ e G4 (0,15 mm), para os solos S1, S2, S3 e S4 
Tabela 2. Determinação de $\mathrm{CaSO}_{4} \cdot 2 \mathrm{H}_{2} \mathrm{O}$ e $\mathrm{CaCO}_{3}$ equivalente, depois da lixiviação das colunas de solo, das amostras de solos $\mathrm{S} 1$, $\mathrm{S} 2$, $\mathrm{S} 3$ e $\mathrm{S} 4$

\begin{tabular}{|c|c|c|c|c|c|c|c|c|c|c|}
\hline \multirow{4}{*}{$\begin{array}{c}\text { Forma } \\
\text { de } \\
\text { Aplicação }\end{array}$} & \multirow{3}{*}{ Mistura } & \multirow{3}{*}{${ }^{00} \mathrm{G}$} & \multicolumn{4}{|c|}{ S1 } & \multicolumn{4}{|c|}{ S2 } \\
\hline & & & \multicolumn{2}{|c|}{$\mathrm{CaSO}_{4} 2 \mathrm{H}_{2} \mathrm{O}$} & \multicolumn{2}{|c|}{$\mathrm{CaCO}_{3}$ Equivalente } & \multicolumn{2}{|c|}{$\mathrm{CaSO}_{4} 2 \mathrm{H}_{2} \mathrm{O}$} & \multicolumn{2}{|c|}{$\mathrm{CaCO}_{3}$ Equivalente } \\
\hline & & & $0-5 \mathrm{~cm}$ & $5-10 \mathrm{~cm}$ & $0-5 \mathrm{~cm}$ & $5-10 \mathrm{~cm}$ & $0-5 \mathrm{~cm}$ & $5-10 \mathrm{~cm}$ & $0-5 \mathrm{~cm}$ & $5-10 \mathrm{~cm}$ \\
\hline & & & \multicolumn{8}{|c|}{$\mathrm{cmol}_{\mathrm{c}} \mathrm{dm}^{-3}$} \\
\hline
\end{tabular}

A.

\begin{tabular}{|c|c|c|c|c|c|c|c|c|c|c|}
\hline \multirow{8}{*}{ Sup. *B1 } & \multirow{4}{*}{${ }^{\circ} \mathrm{C} 1$} & G1 & 0,16 & 0,00 & 0,00 & 0,00 & 0,15 & 0,00 & 0,00 & 0,00 \\
\hline & & $\mathrm{G} 2$ & 0,00 & 0,00 & 0,00 & 0,00 & 0,00 & 0,00 & 0,00 & 0,00 \\
\hline & & G3 & 0,00 & 0,00 & 0,00 & 0,00 & 0,00 & 0,00 & 0,00 & 0,00 \\
\hline & & G4 & 0,00 & 0,00 & 0,00 & 0,00 & 0,00 & 0,00 & 0,00 & 0,00 \\
\hline & \multirow{4}{*}{${ }^{\circ} \mathrm{C} 2$} & G1 & 0,19 & 0,00 & 0,00 & 0,00 & 0,21 & 0,00 & 0,00 & 0,00 \\
\hline & & $\mathrm{G} 2$ & 0,00 & 0,00 & 0,00 & 0,00 & 0,00 & 0,00 & 0,00 & 0,00 \\
\hline & & G3 & 0,00 & 0,00 & 0,00 & 0,00 & 0,00 & 0,00 & 0,00 & 0,00 \\
\hline & & G4 & 0,00 & 0,00 & 0,00 & 0,00 & 0,00 & 0,00 & 0,00 & 0,00 \\
\hline \multirow{8}{*}{ Inc. ${ }^{* *}$ B2 } & \multirow{4}{*}{${ }^{\circ} \mathrm{C} 1$} & G1 & 0,00 & 0,00 & 0,00 & 0,00 & 0,00 & 0,00 & 0,00 & 0,00 \\
\hline & & $\mathrm{G} 2$ & 0,00 & 0,00 & 0,00 & 0,00 & 0,00 & 0,00 & 0,00 & 0,00 \\
\hline & & G3 & 0,00 & 0,00 & 0,00 & 0,00 & 0,00 & 0,00 & 0,00 & 0,00 \\
\hline & & G4 & 0,00 & 0,00 & 0,00 & 0,00 & 0,00 & 0,00 & 0,00 & 0,00 \\
\hline & \multirow{4}{*}{${ }^{\circ} \mathrm{C} 2$} & G1 & 0,00 & 0,00 & 0,00 & 0,00 & 0,00 & 0,00 & 0,00 & 0,00 \\
\hline & & G2 & 0,00 & 0,00 & 0,00 & 0,00 & 0,00 & 0,00 & 0,00 & 0,00 \\
\hline & & G3 & 0,00 & 0,00 & 0,00 & 0,00 & 0,00 & 0,00 & 0,00 & 0,00 \\
\hline & & G4 & 0,00 & 0,00 & 0,00 & 0,00 & 0,00 & 0,00 & 0,00 & 0,00 \\
\hline \multicolumn{11}{|l|}{ B. } \\
\hline \multirow{8}{*}{ Sup. *B1 } & \multirow{4}{*}{${ }^{\circ} \mathrm{C} 1$} & G1 & 0,75 & 0,00 & 0,00 & 0,00 & 0,11 & 0,00 & 0,00 & 0,00 \\
\hline & & G2 & 0,67 & 0,00 & 0,00 & 0,00 & 0,00 & 0,00 & 0,00 & 0,00 \\
\hline & & G3 & 0,33 & 0,00 & 0,00 & 0,00 & 0,00 & 0,00 & 0,00 & 0,00 \\
\hline & & G4 & 0,20 & 0,00 & 0,00 & 0,00 & 0,00 & 0,00 & 0,00 & 0,00 \\
\hline & \multirow{4}{*}{${ }^{\circ} \mathrm{C} 2$} & G1 & 0,82 & 0,00 & 0,04 & 0,00 & 0,12 & 0,00 & 0,00 & 0,00 \\
\hline & & $\mathrm{G} 2$ & 0,69 & 0,00 & 0,03 & 0,00 & 0,00 & 0,00 & 0,00 & 0,00 \\
\hline & & G3 & 0,48 & 0,00 & 0,04 & 0,00 & 0,00 & 0,00 & 0,00 & 0,00 \\
\hline & & G4 & 0,25 & 0,00 & 0,04 & 0,00 & 0,00 & 0,00 & 0,00 & 0,00 \\
\hline \multirow{8}{*}{ Inc. ${ }^{* *} \mathrm{~B} 2$} & \multirow{4}{*}{${ }^{\circ} \mathrm{C} 1$} & G1 & 0,62 & 0,00 & 0,00 & 0,00 & 0,00 & 0,00 & 0,00 & 0,00 \\
\hline & & $\mathrm{G} 2$ & 0,42 & 0,00 & 0,00 & 0,00 & 0,00 & 0,00 & 0,00 & 0,00 \\
\hline & & G3 & 0,21 & 0,00 & 0,00 & 0,00 & 0,00 & 0,00 & 0,00 & 0,00 \\
\hline & & G4 & 0,04 & 0,00 & 0,00 & 0,00 & 0,00 & 0,00 & 0,00 & 0,00 \\
\hline & \multirow{4}{*}{${ }^{\circ} \mathrm{C} 2$} & G1 & 0,66 & 0,00 & 0,04 & 0,00 & 0,00 & 0,00 & 0,00 & 0,00 \\
\hline & & G2 & 0,60 & 0,00 & 0,03 & 0,00 & 0,00 & 0,00 & 0,00 & 0,00 \\
\hline & & G3 & 0,33 & 0,00 & 0,03 & 0,00 & 0,00 & 0,00 & 0,00 & 0,00 \\
\hline & & G4 & 0,10 & 0,00 & 0,04 & 0,00 & 0,00 & 0,00 & 0,00 & 0,00 \\
\hline
\end{tabular}

* B1 aplicado na superfície do solo; ${ }^{* *} \mathrm{~B} 2$ incorporado aos primeiros $5 \mathrm{~cm}$ do solo; ${ }^{\circ} \mathrm{C} 1=100 \%$ de gesso $+0 \%$ de calcário; ${ }^{\circ} \mathrm{C} 2=80 \%$ de gesso $+20 \%$ de calcário; $\mathrm{e}^{\circ 0}{ }^{\circ} \mathrm{G}=$ granulometria do gesso, G1 $(2-1 \mathrm{~mm}), \mathrm{G} 2(1-0,5 \mathrm{~mm}), \mathrm{G} 3(0,5-0,3 \mathrm{~mm})$ e $\mathrm{G} 4(<0,3 \mathrm{~mm})$

PST indicam que, independentemente da forma de aplicação dos corretivos e das granulometrias utilizadas, todas as amostras foram recuperadas quanto à sodicidade (PST $<15$ $\%$ ), tanto para a profundidade de $0-5 \mathrm{~cm}$ como para a profundidade de 5-10 cm. Mustafa \& Abdel-Magid (1981) não observaram efeito significativo da aplicação de gesso na redução da PST de solos altamente salino-sódicos; entretanto, Barros \& Magalhães (1995), trabalhando com solos salinosódicos do Nordeste do Brasil, relataram que a aplicação de gesso corrigiu a PST para valores menores que $15 \%$ para todos os solos estudados. Resultados semelhantes foram obtidos por Silveira (2000), em estudo com solos salino-sódicos de Custódia, PE.

Esses resultados comprovam que a quantidade de gesso determinada em laboratório pelo método de Schoonover modificado (Schoonover M-1) é adequada e que o método pode ser utilizado para correção dos solos salino-sódicos em estudo. A redução da PST, mesmo de um solo que apresenta uma PST $>80$ \% (amostra S3), é explicada pela relação entre a PST do solo e a dissolução do gesso. Esta relação foi demonstrada por diversos pesquisadores, entre eles Hira \& Singh (1980), que relataram que a concentração de sulfato na solução eluída de colunas de solo tratadas com gesso aumentou, juntamente com a PST do solo, indicando maior solubilidade do gesso a altos valores de PST $(\mathrm{PST}=94 \%)$.

A influência da PST sobre a dissolução do gesso provavelmente pode ser indireta, o que, na verdade, pode estar controlando a taxa de dissolução do gesso é a presença de sais de sódio dissolvidos na solução do solo. A aplicação de gesso em solos salino-sódicos tende a elevar a solubilidade do gesso, 
A.

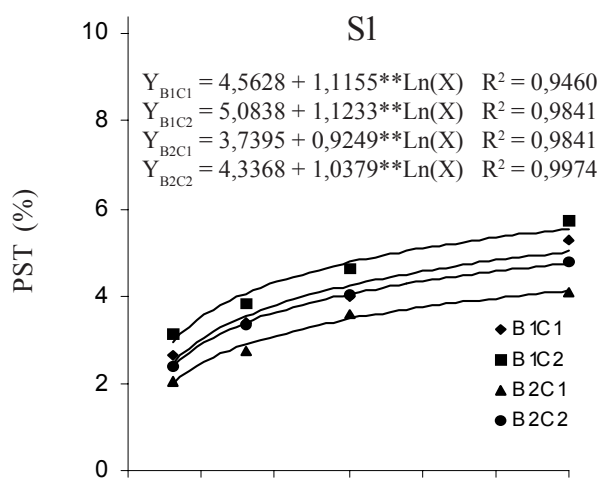

B.

C.

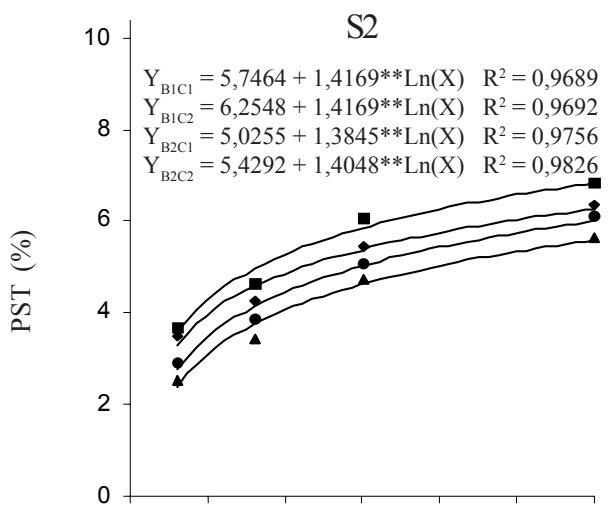

- B 1C1

- B 1C2

$\triangle \mathrm{B} 2 \mathrm{C} 1$

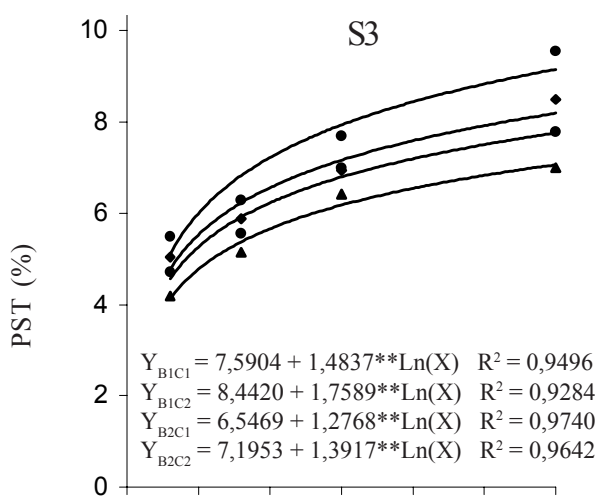

D.

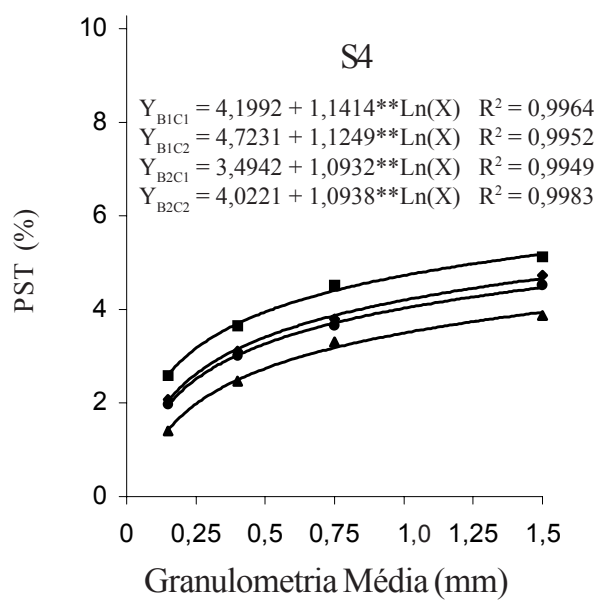

Figura 3. Percentagem do sódio trocável na profundidade de $0-5 \mathrm{~cm}$ do solo, em relação à forma da aplicação dos corretivos na superfície (B1), incorporado (B2), mistura C1 (100\% de gesso + $0,00 \%$ de calcário e mistura $\mathrm{C} 2(80 \%$ de gesso $+20 \%$ de calcário), em função da granulometria média do gesso (G), G1 (1,5 mm),G2 $(0,75 \mathrm{~mm}), \mathrm{G} 3(0,4 \mathrm{~mm}) \mathrm{eG} 4(0,15 \mathrm{~mm})$, para os solos S1, S2, S3 eS4
A.

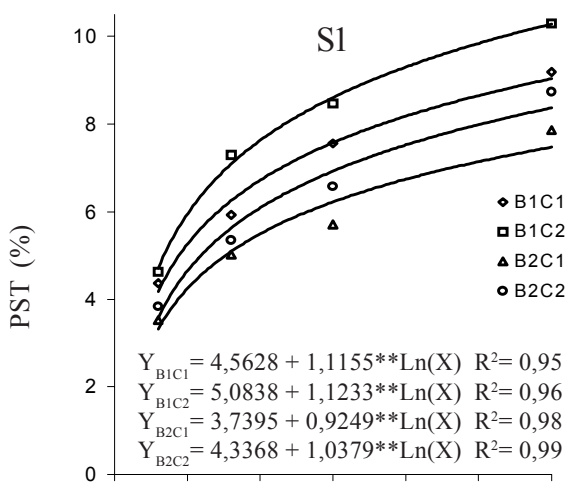

B.

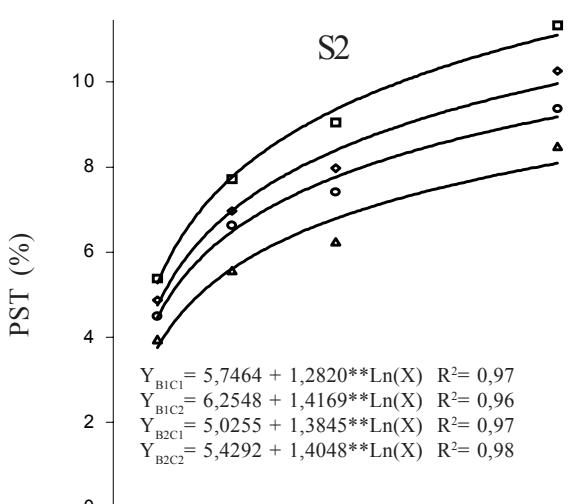

- B1C1

C.

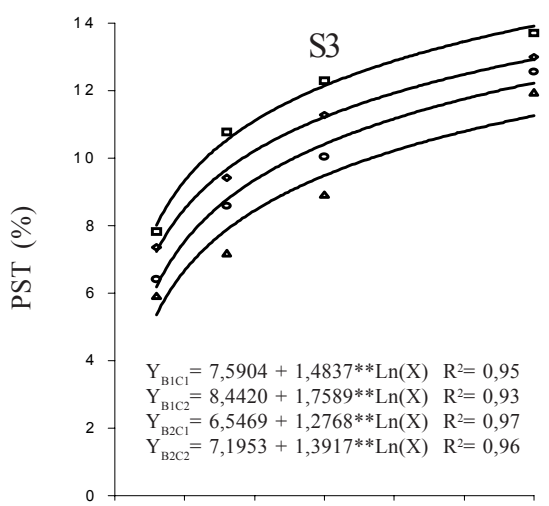

D.

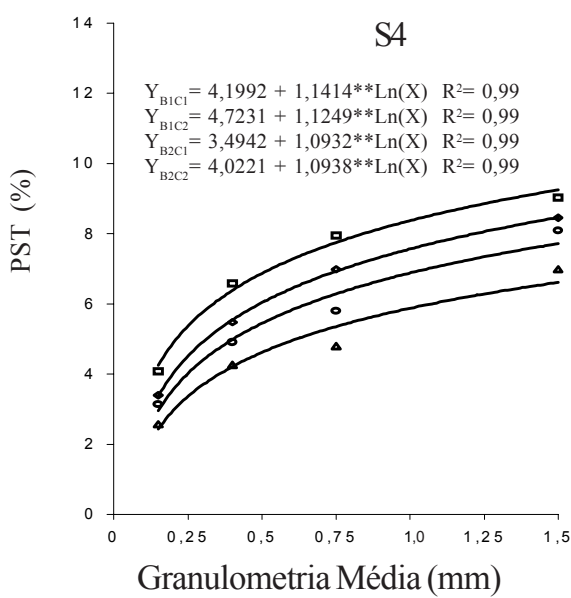

Figura 4. Percentagem do sódio trocável na profundidade de 5-10 $\mathrm{cm}$ do solo, em relação a forma da aplicação dos corretivos na superficie (B1), incorporado (B2), mistura C1 (100\% de gesso + $0,00 \%$ de calcário) e mistura $\mathrm{C} 2$ ( $80 \%$ de gesso $+20 \%$ de calcário), em função da granulometria média do gesso (G), G1 (1,5 mm), G2 (0,75 mm), G3 (0,4 mm)e G4 (0,15 mm), paraos solos S1, S2, S3 eS4. 
e este aumento é proporcional à concentração de íons de $\mathrm{Na}+$ e Cl- na solução do solo (Richards, 1954; Santos, 1995).

\section{CONCLUSÕES}

1. A quantidade de gesso determinada em laboratório pelo método de Schoonover modificado (Schoonover M-1) é adequada ao deslocamento do sódio trocável do complexo de troca.

2. A eficiência do gesso e da mistura gesso + calcário na recuperação dos solos salino-sódicos, mostra-se superior quando os corretivos são incorporados aos primeiros $5 \mathrm{~cm}$ das colunas de solo.

3. Os tratamentos relativos às granulometrias de gesso mais finas G3 $(0,5-0,3 \mathrm{~mm})$ e G4 $(<0,3 \mathrm{~mm})$, apresentaram melhor desempenho na substituição do sódio trocável.

\section{LITERATURA CITADA}

Ahamad, E.; Jones, R.L.; Hinesly, T.D. Electromelioration of a sodic horizon from an Illinois Natraqualf. Soil Science Society of America Journal, Madison, v.61, p.1761-1765, 1997.

Alcordo, I.S.; Rechcigl, J.E. Phosphogypsum in agriculture. A Review. Advances in Agronomy, New York, v.118, p.49-55, 1993.

Barros, M.F.C.; Magalhães, A.F. Avaliações de métodos de determinação da necessidade de gesso em solos salinosódicos. Revista Brasileira de Ciência Solo, Campinas, v.13, p.119-123, 1989.

Barros, M.F.C.; Magalhães, A.F. Recuperação de solos salinosódicos II. Eficiência do gesso aplicado. In: Congresso Brasileiro de Ciência do Solo, 25. 1995. Viçosa. Resumos... Viçosa: SBCS, p.2263-2265.

de Jong, E. Reclamation of solis contaminated by sodium chloride. Canadian Journal Soil Science, Ottawa, v.62, p.351364, 1982.

EMBRAPA - Empresa Brasileira de Pesquisa Agropecuária. Manual de métodos de análises de solo 2, ed. Rio de Janeiro: EMBRAPA, 1997.212p.

Hira, G.S.; Singh, N.T. Irrigation water requirement for dissolution of gypsum in sodic soil. Soil Science Society of America Journal, Madison, v.44, p.930-933, 1980.
Holanda, J.S.; Vitti, G.C.; Salviano, A.A.C. Alterações nas propriedades químicas de um solo aluvial salino-sódico decorrentes da subsolagem e do uso de condicionadores. Revista Brasileira de Ciência do Solo, Campinas, v.22, p.387394, 1998.

Liang, J.; Karamanos, R.E.; Moir, M.E. The influence of brine contamination and phosphogypsum amendments on soil chemical properties and plant response. Soil Science Plant Analysis, New York, v.26, p.1033-1057, 1995.

Magalhães, A.F. Manejo da fertilidade dos solos irrigados: produtividade, degradação e correção. In: Reunião Brasileira de Fertilidade do Solo e Nutrição de Plantas, 21, 1995, Petrolina. Anais... Petrolina: SBCS, 1995. p.77-86.

McKenzie, D.C.; Abbott, T.S.; Chan, K.Y.; Slavich, P.G.; Hall. D.J.M. The nature, distribution and management of sodic soils in New South Wales. In: Naidu, R.; Sumner, M.E.; Rengasamy, P. Australian sodic soils: distribution, properties and management. Victoria: CSRIO, 1995. p.246-264.

Mustafa, M.A.; Abdel-Magid, E.A. The effects of irrigation interval, urea-N and gypsum on salt redistribution in a highly saline-sodic montmorillonitic clay soil under forage sorghum. Soil Science, Baltimore, v.132, p.308-15, 1981.

Pereira, J.R.; Valdivieso, C.R.; Cordeiro, G.G. Recuperação de solos afetados por sódio através do uso de gesso. In: Seminário sobre o Uso de Fosfogesso na Agricultura, 1, 1985, Brasília. Anais..., Brasília: IBRAFOS, 1986. p.85-105.

Qadir, M.; Qureshi, R.H.; Ahmad, N. Reclamation of a salinesodic soil by gypsum and Leptochloa fusca. Geoderma, Amsterdam, v.74, p.207-217, 1996.

Ramirez, H.; Rodriguez, O.; Shainberg, I. Effect of gypsum on furrow erosion and intake rate. Soil Science, Baltimore, v.164, p.351-357, 1999.

Richards, L.A. Diagnosis and improvement of saline and alkali soils. Washington: US Department of Agriculture, 1954. 160p. USDA Agricultural Handbook, 60

Sampaio, R.A. Manejo da água na recuperação de solos salinosódicos, em colunas. Viçosa: UFV, 1993, 75p. Dissertação Mestrado

Santos, R.V. Correção de um solo salino-sódico e absorção de nutrientes pelo feijoeiro vigna (Vigna unguiculata (L.) WALD). Piracicaba: USP, 1995. 120p. Tese Doutorado

Silveira, K.R. Influência da aplicação do gesso na água de irrigação sobre a salinidade, sodicidade e condutividade hidráulica de solos aluviais. Recife: UFRPE, 2000. 73p. Dissertação Mestrado 